\title{
Characterizing Texture and Grain Boundaries in Nanoscale Cu Interconnects by Precession Electron Diffraction
}

\author{
K. J. Ganesh*, A. Darbal**, S. Rajasekhara*, G. S. Rohrer**, K. Barmak**, P. J. Ferreira* \\ * Materials Science and Engineering Program, University of Texas at Austin, Austin, TX 78712 \\ ** Materials Research Science and Engineering Center, Department of Materials Science and \\ Engineering, Carnegie Mellon University, 5000 Forbes Avenue, Pittsburgh, PA 15213
}

The constant downscaling of back-end of line $\mathrm{Cu}$ interconnects (CIs) has resulted in changes to their microstructure [1]. Among these changes, any variation in local texture and grain boundary types could strongly affect reliability issues like stress migration and electromigration $[2,3]$. In the current work, we couple precession electron microscopy and D-STEM [4] using the ASTAR system from NanoMEGAS to obtain texture information in $180 \mathrm{~nm}$ and $120 \mathrm{~nm}$ wide damascene $\mathrm{Cu}$ lines with a spatial resolution of 1-2 $\mathrm{nm}$. Furthermore, we perform misorientation and trace analysis using the TSL OIM software to investigate the presence of $\Sigma 3$ boundaries, which are typically predominant in $\mathrm{Cu}$, and non-CSL high angle boundaries [5].

The microstructure of both $120 \mathrm{~nm}$ and $180 \mathrm{~nm}$ CIs mainly comprises large bamboo-type grains. However, in the case of the $120 \mathrm{~nm}$ CIs, clusters of small grains in the vicinity of large bamboo-type grains can be observed (Fig 1a). The change in microstructure with line width for $\mathrm{Cu}$ is similar to earlier observations in Al interconnects [6]. Representative plan-view color coded inverse pole figure maps from these lines are shown in Figs. 1a and 2a. $120 \mathrm{~nm}$ interconnect specimens reveal a slight $<111>$ fiber texture along the width of the line (RD) as shown in Fig. 1b. This can be attributed to a dominant sidewall growth of (111) grains in narrower line widths and is consistent with previous work [7]. On the other hand, $180 \mathrm{~nm}$ interconnects show a prominent bi-axial texture of $<110>\| \mathrm{RD}$ and $<111>\| \mathrm{ND}$ (Fig.2b). Orientation maps from multiple lines in cross section (Fig. 2c) clearly depict bottom-up growth of grains with a preferred $<110>$ texture along the RD. Grain boundary trace analysis carried out on data sets of 6104 boundaries for $120 \mathrm{~nm}$ lines and 5780 boundaries for $180 \mathrm{~nm}$ wide lines showed that more than $90 \%$ of the $\Sigma 3$ twin boundaries in the lines are coherent in nature. The length fraction of coherent $\Sigma 3$ boundaries was found to be $24 \%$ for 180 $\mathrm{nm}$ lines and $20 \%$ for $120 \mathrm{~nm}$ lines.

The orientation map also reveals that in the case of $120 \mathrm{~nm}$ CIs, groups of small grains, such as those in Fig.1a are separated by high angle boundaries. The clusters of non-bamboo type small grains in $120 \mathrm{~nm}$ lines may raise the level of local thermal stresses and the high angle boundaries separating them may provide fast diffusion paths for mass transport to influence stress migration reliability in interconnect lines. Triple junctions connecting such boundaries may also act as flux divergence sites and influence electromigration reliability $[3,8]$. The aforementioned technique and analysis can be extended to smaller state of the art $\mathrm{Cu}$ lines to study their microstructure and correlate that with the reliability of $\mathrm{Cu}$ interconnects.

References:

[1] J. Paik et al., J. Appl. Phys. 99 (2006) 024509-1.

[2] J. A. Nucci et al., Appl. Phys. Lett. 70 (1997) 1242.

[3] P. S. Ho et al., Int. J. of Mater. Res. 101 (2010) 216. 
[4] K. J. Ganesh et al., Microsc. Microanal. 16 (2010) 614.

[5] S. I. Wright et al., J. Microsc. 205 (2002) 245.

[6] B. D. Knowlton et al., J. Appl. Phy. 81 (1997) 6073.

[7] P. Besser et al., J. Electron. Mater. 30 (2001) 320 and K. J. Ganesh et al., Scr. Mat. 62 (2010) 843.

[8] E. Zschech et al., Pro. Adv. Metallization Conf. (2002) 305.

[9] AD, GSR and KB acknowledge financial support of the MRSEC program NSF DMR-0520425.

[10] KJG, PJF acknowledge financial support from Semiconductor Research Corporation, Task id2072.001 .

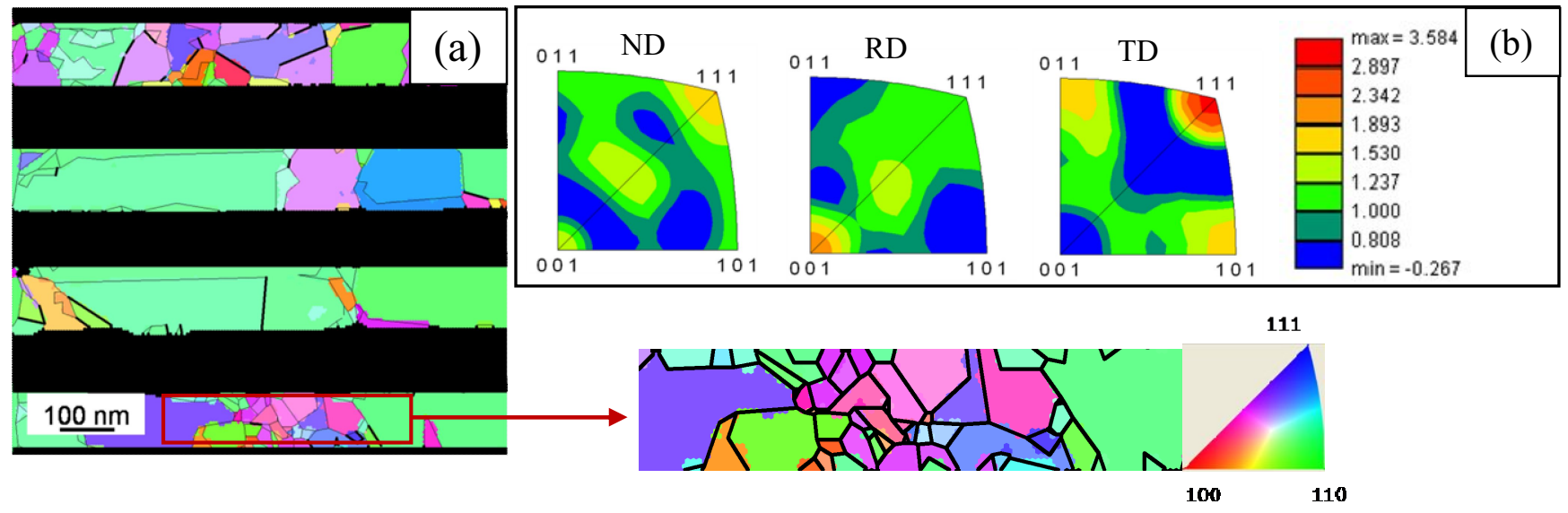

Fig. 1: (a) Color coded inverse pole figure (IPF) map from $120 \mathrm{~nm}$ wide $\mathrm{Cu}$ lines. The boundaries marked with thick, dark lines are coherent $\Sigma 3$ boundaries while those marked by thin lines are non CSL high angle boundaries with misorientation angle greater than $20^{\circ}$. A magnified image of a cluster of small grains is shown on the right along with the color code for the IPF (b) Inverse pole figure plots along the normal direction (ND), perpendicular to the sample plane, rolling direction (RD), along the length of the line and transverse direction (TD), along the width of the line. A slight $<111>$ fiber texture is seen along TD.

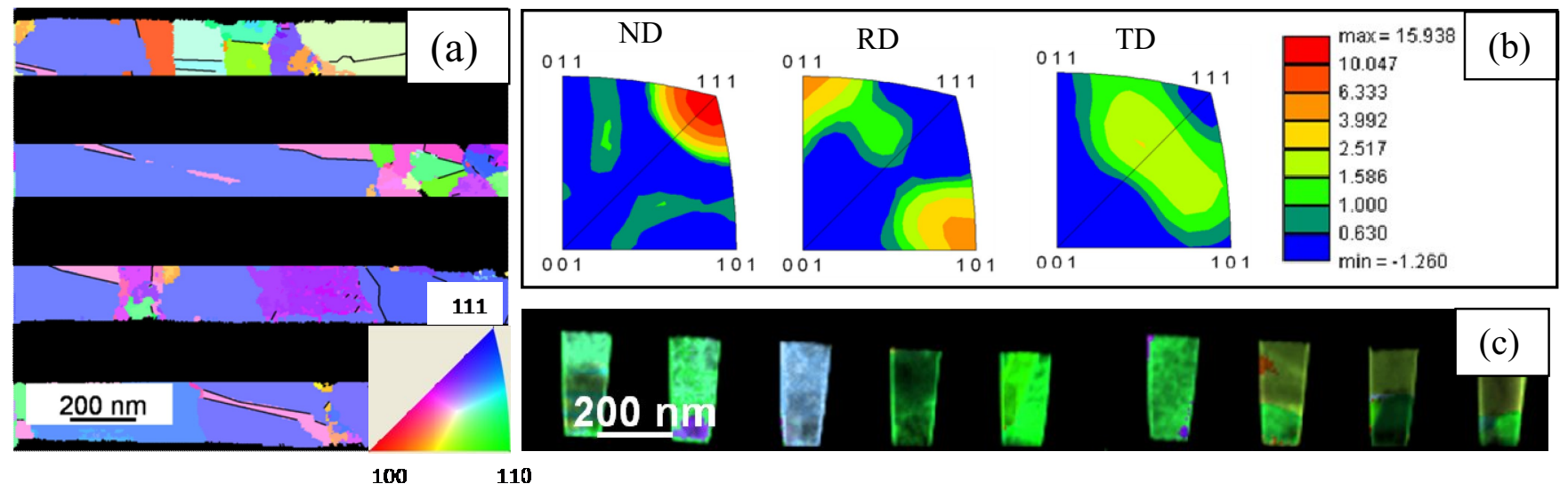

Fig. 2: (a) Color coded IPF map from $180 \mathrm{~nm}$ wide $\mathrm{Cu}$ lines. Coherent $\Sigma 3$ boundaries are marked by thick, dark lines. Inset shows the color codes for the IPF (b) Transverse cross section IPFs overlaid on the virtual dark field image (c) Inverse pole figure plots along the normal direction (ND), perpendicular to the sample plane, rolling direction (RD), along the length of the line and transverse direction (TD), along the width of the line. A strong bi-axial texture is observed with $<111>\|$ ND and $<110>\| \mathrm{RD}$. 